\title{
Mathieu TERRIER, Sepideh PARSAPAJOUH (dir.), "Cimetières et tombes dans les mondes musulmans. À la croisée des enjeux religieux, politiques et mémoriels »
}

Revue des mondes musulmans et de la Méditerranée, 146, 2019.

\section{Catherine Mayeur-Jaouen}

\section{OpenEdition}

Journals

Édition électronique

URL : https://journals.openedition.org/assr/65559

DOI : $10.4000 /$ assr.65559

ISSN : $1777-5825$

Éditeur

Éditions de l'EHESS

Édition imprimée

Date de publication : 4 décembre 2021

Pagination : 431-434

ISBN : 9782713228735

ISSN : 0335-5985

\section{Référence électronique}

Catherine Mayeur-Jaouen, « Mathieu TerRier, Sepideh parsapajoun (dir.), « Cimetières et tombes dans les mondes musulmans. À la croisée des enjeux religieux, politiques et mémoriels » », Archives de sciences sociales des religions [En ligne], 196 | octobre-décembre 2021, mis en ligne le 01 décembre 2021, consulté le 13 février 2022. URL : http://journals.openedition.org/assr/65559 ; DOI : https:// doi.org/10.4000/assr.65559

Ce document a été généré automatiquement le 13 février 2022.

(c) Archives de sciences sociales des religions 


\section{Mathieu TERRIER, Sepideh}

PARSAPAJOUH (dir.), « Cimetières et tombes dans les mondes musulmans. À la croisée des enjeux
religieux, politiques et mémoriels »

Revue des mondes musulmans et de la Méditerranée, 146, 2019.

\section{Catherine Mayeur-Jaouen}

\section{RÉFÉRENCE}

Mathieu TERRIER, Sepideh PARSAPAJOUH (dir.), « Cimetières et tombes dans les mondes musulmans. À la croisée des enjeux religieux, politiques et mémoriels ", Revue des mondes musulmans et de la Méditerranée, 146, 2019.

Le numéro 146 de la REMMM, paru en 2019, comprend un numéro thématique et trois études libres. Nous ne traiterons ici que du numéro thématique qui porte sur les cimetières et les tombes dans les mondes musulmans (p.11-195). L'introduction (p. 11-24), écrite par Sepideh Parsapajouh et Mathieu Terrier, rappelle l'importance anthropologique et historique de l'inhumation. "Aussi les cimetières et les tombes constituent-ils depuis longtemps des objets privilégiés pour les sciences humaines, sociales et historiques", comme "l'un des lieux où, par excellence, se construisent la mémoire collective des individus et la mémoire culturelle d'une société ». Les cimetières, inscrits dans le système religieux, témoignent « des croyances relatives au destin de l'âme dans l'au-delà comme des pratiques rituelles définissant la communauté dans le monde ». En islam, religion du salut pour laquelle la mort est un passage de la vie d'ici-bas à l'au-delà, le cimetière occupe une place considérable, à la fois lieu de sociabilité, de rencontre et «interface» entre le monde des vivants et le monde des 
morts - situé dans l'horizon de la résurrection. Certains cimetières devenus d'immenses nécropoles ont entraîné la création de villes entières. L'introduction mentionne brièvement le lien fondamental avec le culte des saints musulmans - soufis ou chiites -, parfois remplacé par le culte des martyrs (p. 14 et note 6). Il faudrait rappeler que l'édification de mausolées, dans le sunnisme, et la visite des tombes ne sont pas une pratique exclusivement soufie, et que l'omniprésence du soufisme dans les dévotions aux tombes ne remonte pas en amont $\mathrm{du} \mathrm{xIII}^{\mathrm{e}}$ siècle. Ce furent souvent d'autres causes qui motivèrent les dévotions aux tombes et aux cimetières, $d u v{ }^{e}$ au $\mathrm{XIII}^{\mathrm{e}}$ siècle, comme la concurrence entre chiisme et sunnisme et les facteurs politiques (cultes dynastiques).

2 Le dossier étudie le fait funéraire dans les mondes musulmans d'aujourd'hui, et au-delà, dans les diasporas et migrations contemporaines, en conjuguant les approches de l'islamologie, des sciences sociales et de l'histoire culturelle. Trois points de vue sont privilégiés: conception $\mathrm{du}$ monde et $\mathrm{du}$ destin individuel dans le fait religieux funéraire ; enjeux politiques de l'inhumation et de la gestion des cimetières ; mémoire culturelle. Le premier enjeu sera le moins développé (un seul article dû à Mathieu Terrier) ; le second, beaucoup mieux traité, aborde le contrôle du funéraire par les États qui vont «de la répression à la patrimonialisation, en passant par la bureaucratisation » (p. 14), les cimetières étant surinvestis de symboles politiques; le troisième, à propos de la formation de l'identité collective, est le plus approfondi. Trois phénomènes principaux sont étudiés: le passage de sociétés musulmanes sous «l'autorité de régimes socialistes hostiles à la tradition religieuse [la Chine, l'URSS] ; [la] mondialisation de l'islam; [la] nouvelle politisation de l'islam depuis les années 1970, notamment dans le monde chiite. »

3 La première partie est intitulée « Croyances et pratiques funéraires entre orthodoxie et hétérodoxie ». Cet intitulé un peu gênant reprend des termes qui n'ont de sens que pour certains musulmans et dans certains horizons, de façon d'ailleurs différente selon les époques. Il est juste d'écrire dans l'introduction qu'il existe « un consensus profond sur la légalité et la valeur éthique de la visite aux tombeaux [et sur] le sort des défunts outre-tombe [qui] réunit le soufisme, le chiisme et la philosophie islamique » et moins exact d'écrire, sans préciser, que ces «trois courants [sont] hâtivement perçus comme hétérodoxes et marginaux ». Mais perçus quand, par qui et par rapport à quoi ? Il faut rappeler que les «prescriptions [qui] se réclament de la shari'a» (p.16) ne sont rien d'autre que du hadith, lui-même objet de collections variées, contradictoires, et interprétées par toute sorte de commentaires et de classements, chez les chiites comme chez les sunnites. Pour parler de la tombe dans le hadith (un sujet fondamental), on trouvera les ouvrages ou articles de référence dus à Walter Diem et à Marco Schöller, ainsi qu'à Thomas Leisten et à Leor Halevi.

4 La première partie comprend deux articles: d'abord une longue et intéressante contribution de Mathieu Terrier, la seule qui soit fondée sur des sources textuelles islamiques et qui aborde les questions doctrinales auxquelles les autres articles se contentent de faire allusion. « La tombe comme isthme (barzakh) entre les vivants et les morts » compare les écrits du grand écrivain sunnite et soufi Ghazâlî (m. 1111) dans l'Ihyâ' 'ulûm al-dîn et leur reprise par un chiite duodécimain, Kâshânî (m. 1680). Terrier souligne l'importance de la réflexion théologique sur ces questions, et une vraie convergence entre les deux points de vue: la visite des tombes est une source d'édification morale et spirituelle pour les vivants. Saluons cette comparaison, trop 
rare, entre textes chiites et sunnites, en regrettant toutefois qu'elle ne confronte pas deux textes de même époque. Même si Ghazâlî a été lu continûment et que les lectures de l'Ihyâ' en font un texte toujours actuel, il aurait sans doute mieux valu prendre un commentaire sunnite de l'Ihyâ' au XVII ${ }^{\mathrm{e}}$ ou XVIII ${ }^{\mathrm{e}}$ siècle (celui de Murtadâ al-Zabîdî par exemple) ou tout texte ottoman sur ces questions pour avoir une pleine équivalence historique entre les deux termes de la comparaison. Faute de quoi l'absence d'historicisation laisse penser que Ghazâlî résumerait une fois pour toutes les attitudes soufies et sunnites, et pour des siècles, alors qu'il écrit avant Ibn 'Arabî et avant la mise en place des grandes confréries - acteurs importants de la structuration des cimetières comme lieux dévotionnels; avant aussi Ibn Taymiyya au début du XIv siècle ; avant le wahhabisme du XVIII ${ }^{e}$ siècle, surtout. Au moment où écrit Kâshânî, le culte des saints et les dévotions aux tombes dans le sunnisme ottoman venaient justement de traverser leur première vraie crise face aux Kadizadeli, influents au cœur de l'Empire - auxquels s'opposa Nâbulsî (1641-1731), soufi akbarien et apologue des visites aux tombes.

Delphine Ortis décrit avec clarté et sobriété plusieurs espaces funéraires à Sehwan Sharîf, ville de pèlerinage du Sindh pakistanais, autour du tombeau de Lal Shahbâz Qalandar (m.1274) : son terrain mené sur plusieurs années dans les cimetières publics (qabristân) et la vingtaine d'hospices soufis (kâfî) construits dans la ville autour des tombes des disciples-compagnons du saint lui permet de discerner des évolutions, notamment le passage de tombes «crues» à des tombes "cuites» en matériaux durables. Elle signale " que les différents traitements matériels de la tombe ici-bas sont le miroir d'une destinée différenciée des morts dans l'au-delà » (p.47), reflet de leur statut dans l'ici-bas. À propos de Lâl Shahbâz, reprenant un article d'Emma AubinBoltanski, Delphine Ortis remarque à juste titre que «le sépulcre constitue en quelque sorte le corps du saint ». Il s'agit en effet le plus souvent de cénotaphes, ce qui serait à approfondir. Trait particulièrement intéressant, elle signale la présence toujours actuelle de renonçants installés parmi les tombes auxquelles ils donnent vie par des récits de rêve ou de miracles. Entre soufisme et chiisme, grâce aux qalandars, se perpétue une mémoire religieuse qui gravite autour du sanctuaire (dargâh) de Lâl Shahbâz Qalandar.

6 La deuxième partie porte sur l'islam en contexte socialiste, soviétique et chinois : " entre hostilité, indifférence et volonté de récupération », les régimes socialistes ont vu les pratiques funéraires s'affirmer comme « des lieux de résistance et de résilience de l'identité ». Marie-Paule Hille montre les changements advenus dans une petite ville musulmane du Gansu, au nord-ouest de la Chine, entre 1968 et 2018 (mais surtout depuis le début des années 2010), où la communauté musulmane chinoise, d'origine soufie, du Xidaotang parvient difficilement à maintenir les tombes de ses saints (ses quatre maîtres défunts), dans un processus de patrimonialisation. Celui-ci, qui accompagne une évolution urbaine rapide (" projet pharaonique » de constructions par le Xidaotang, compétition religieuse, reconfiguration de l'espace urbain), est menacé par les critiques des mouvements réformistes, mais surtout par les contraintes politiques qui obligent le mouvement à afficher sa loyauté politique. Extrêmement bien documenté, l'article comprend des photographies et schémas.

7 Isabelle Ohayon revient à la vénération des morts en général, en l'étudiant dans l'islam kazakh à l'époque soviétique : de 1960 à 1980, un moment de relatif relâchement des contraintes permet d'exploiter un lopin de terre ou un cheptel face à une politique religieuse de contrôle, d'institutionnalisation et de répression qui a modifié les 
pratiques funéraires. Les pénuries et restrictions de l'économie soviétique constituent un défi matériel pour les familles désireuses d'organiser de dignes funérailles, mais l'assouplissement du "soviétisme tardif» permet d'augmenter les dépenses (dons, distributions d'étoffes et d'argent, repas, émolument du mollah) lors des cérémonies et favorise la présence de mollahs clandestins itinérants, notamment naqshbandis, de plus en plus nombreux. Les contraintes sur les visites pieuses (ziyârât) régionales aux lieux saints d'Asie centrale (dont le tombeau de Baha al-Dîn Naqshband) ont reporté les vénérations des fidèles vers les cimetières ordinaires - espace d'autonomie relative, d'où l'essor de la construction de mausolées pour les figures d'autorité locale au Kazakhstan des années 1970.

8 La troisième partie, "Reterritorialiser la mort », explore les pratiques de l'inhumation hors du "dâr al-islâm»-les auteurs utilisent ici un terme médiéval pour décrire les États où l'islam est religion d'État et où la majorité de la population est musulmane, mais il faudrait préciser qu'une grande majorité de musulmans n'utilisent pas cette terminologie musulmane. Il est question du cimetière musulman de Guarulhos à Sao Paulo (Michelangelo Giampaoli) et du Grand Londres (Nada Afiouni). Dans les deux cas, s'affirme le choix d'inhumer sur place plutôt que de rapatrier le corps dans le pays d'origine - que parfois les défunts n'ont qu'à peine connu. Les raisons de ce choix peuvent être économiques, citoyennes ou doctrinales - selon les normes du "néohanbalisme »- et donc affirmer aussi bien l'acclimatation d'un islam brésilien en contexte multiculturel que l'affirmation d'une spécificité islamique, voire salafiste. À Sao Paulo, l'étude de l'espace funéraire permet de reconstituer l'histoire et la mémoire d'une partie de la communauté musulmane brésilienne. À Londres, "la création et l'essor fulgurant du premier cimetière privé musulman au Royaume-Uni [sont] avant tout l'histoire d'une réussite commerciale » (p. 152).

9 La quatrième partie porte sur les cimetières chiites contemporains avec deux terrains fort bien traités par des spécialistes chevronnées: les enjeux mémoriels dans les cimetières villageois du Sud-Liban rural sont présentés par Kinda Chaib, à propos des martyrs enterrés dans ces cimetières - soit mis en avant par le Hezbollah, soit invisibilisés. Mais les histoires oubliées affleurent dans ces cimetières, par strates superposées sous l'hégémonie mémorielle apparente - ce qui permet une histoire sociale du Liban rural. Un impressionnant article de Sepideh Parsapajouh, abondamment illustré, présente enfin le grand cimetière de Téhéran (Behesht-e Zahra) qui s'étend sur 700 à 800 hectares (les deux chiffres sont mentionnés), avec 200 enterrements par jour en moyenne. Se jouent sur le terrain - une «nécropole hiérarchisée » avec ses divisions spécialisées - l'influence de la rationalité de l'État, les normes rituelles définies par les religieux et suivies par l'administration funéraire, et une organisation placée sous l'égide de la municipalité de Téhéran, mais aussi sous l'influence de l'institution cléricale et de la Fondation des martyrs, et aussi de la Maison de l'ayatollah Khomeiny qui s'occupe de son mausolée. Finalement, malgré tout, se font jour les pratiques «des simples croyants attachés au culte des morts» (p. 173.) Ce dernier article rejoint de précédentes publications de Sepideh Parsapajouh, pour composer une œuvre convaincante sur les rites funéraires en évolution dans l'Iran contemporain.

10 Il faut saluer ce numéro pionnier de la REMMM inscrit dans la série Monde contemporain, et dû, pour l'essentiel, à des anthropologues qui proposent un tableau actuel, large et au fait des évolutions récentes, sur un sujet fondamental. On peut regretter un peu que 
la bibliographie de fond proposée reste francophone sur des sujets où les livres récents sont plutôt en allemand ou en anglais; inversement, il est dommage que le célèbre article d'Ignaz Goldziher soit cité dans sa traduction anglaise datée de 1971 (note 3 p. 74), sans que le lecteur non prévenu puisse se douter qu'il a été publié en français en 1880 dans la Revue de l'histoire des religions (il est donc disponible sur Gallica d'une part, et a été récemment réédité d'autre part). Cet article n'est pas une «réflexion complète sur le culte des saints en islam ", mais un point de vue réformiste prowahhabite dont l'érudition et le brillant ont beaucoup influencé l'historiographie occidentale, l'orientalisme puis le réformisme musulman. Pour Goldziher, certes remarquable source d'inspiration, le wahhabisme était le véritable islam, et le culte des saints, pour l'essentiel, un agrégat de survivances païennes. Il est question aussi des "normes théologico-juridiques du néo-hanbalisme » qu'il importe de définir : cette expression, reprise de Henri Laoust, évoque Ibn Taymiyya (m.1326), peut-être les wahhabites. Il faut rappeler que les visites aux tombes ne sont pas par elles-mêmes condamnées par Ibn Taymiyya, soufi qâdirî, qui encourage les ziyârât à des fins édifiantes, du moment qu'il n'y a aucun pèlerinage.

Le livre convainc le lecteur de la variété et de la réalité des pratiques funéraires et des enjeux sociaux et mémoriaux qui se jouent autour des cimetières, et surtout du culte des tombes. Le volume remplit son objectif : oui, les cimetières ont encore beaucoup à nous apprendre sur l'islam, sur le culte des saints, sur l'histoire sociale entre local et global, sur les rapports à la mort et au sacré, sur les formes de sécularisation et son rapport au politique. En partant de ce tableau, et en utilisant les nombreux travaux en cours sur les cimetières en islam à la fin du Moyen Âge et à l'époque moderne ( $\mathrm{Xv}^{\mathrm{e}}$ XVIII siècle), on pourra dans l'avenir préciser sur la longue durée où et comment s'expriment les adversaires des visites aux tombes et du culte des morts: un nouvel objectif en perspective. 